\title{
Active Helicobacter pylori Infection Is a Risk Factor for Colorectal Mucosa: Early and Advanced Colonic Neoplasm Sequence
}

\author{
Jannis Kountouras, Nikolaos Kapetanakis, Stergios A. Polyzos, Panagiotis Katsinelos, Emmanuel Gavalas, Dimitri Tzivras, \\ Christos Zeglinas, Constantinos Kountouras, Elizabeth Vardaka, Eyripidis Stefanidis, and Evagelos Kazakos
}

Department of Gastroenterology, Second Medical Clinic, Ippokration Hospital, Aristotle University of Thessaloniki, Thessaloniki, Greece

\section{To the Editor:}

Based on serology, Lee et al. ${ }^{1}$ concluded that Helicobacter pylori infection (Hp-I) increased the risk of advanced colorectal neoplasm (CRN), particularly when combined with atrophic gastritis (AG), thereby warranting strict colonoscopy screening and surveillance in $\mathrm{Hp}$-positive AG patients. Indeed, $\mathrm{Hp}$-related chronic gastritis could be involved in an increased risk of CRN that seems to be enhanced by the progression of gastric atrophy and the occurrence of active inflammation. ${ }^{2}$

However, the serological test does not accurately discriminate between current and past infections, also mentioned by the aurhors, ${ }^{1}$ and, apart from past infection that might even be more relevant for oncogenesis, such a distinction is crucial because only current $\mathrm{Hp}$-I induces humoral and cellular immune responses that induce or perpetuate chronic inflammatory processes in gastrointestinal tract with potential oncogenic sequelae; many neoplasms including colorectal carcinoma (CRC) arise at the sites of chronic inflammation and infection., ${ }^{3,4}$

Based on histology, the practical gold standard for current $H p-I$ diagnosis, our data in 50 CRC patients, 25 patients with colorectal adenomas (CRA) and 10 controls, showed significantly higher presence of $\mathrm{Hp}$-I in the CRA (68\%) and CRC (84\%) groups compared with controls (30\%). ${ }^{5}$ Regarding the features of histological severity in CRA group, presence of $\mathrm{Hp}$-I was observed in 50\% of patients with mild and $80 \%$ of patients with moderate/severe dysplasia. Likewise, presence of $\mathrm{Hp}$-I in the CRC group was observed in 89\% of patients with mild and 83\% of patients with moderate/severe grade. ${ }^{5}$ Noteworthy, Hp presence was documented by immunohistochemical stain in CRA and CRC tissues. ${ }^{5}$ In addition, presence of $\mathrm{Hp}$-I with accompanying immunohistochemical expression of CD44 (indicator of cancer stem cells [CSCs] and/or bone marrow-derived stem cells
[BMDSCs]) in biopsy specimens was found in a high proportion of CRA patients accompanied with moderate/severe dysplasia (88\%) and CRC patients with moderate/severe degree of malignancy (91\%)., ${ }^{5,6}$ Comparable pictures were also obtained for proliferation marker Ki-67, anti-apoptotic Bcl-2 and CD45 (assessing mainly $\mathrm{T}$ and $\mathrm{B}$ lymphocytes locally) immunohistochemical expressions. ${ }^{5,6}$

By introducing univariate analysis, the authors found that metabolic syndrome (MetS) was significantly associated with overall and advanced CRN. ${ }^{1}$ In this regard, in a systematic review, we reported an association between $\mathrm{Hp}$-I and insulin resistance (IR), the major underlying mechanism responsible for the MetS. ${ }^{7}$ Our data further indicate that $\mathrm{Hp}$-I might represent one further hit contributing to nonalcoholic fatty liver disease (NAFLD) pathogenesis, representing the hepatic component of MetS ${ }^{8}$ NAFLD closely related to IR is involved in colon oncogenesis. Other studies also suggest that $\mathrm{Hp}$-I with concomitant MetS might further increase CRA risk. ${ }^{9}$

Components of MetS are also associated with esophageal adenocarcinoma (EAC) risk and $\mathrm{Hp}$-related $\mathrm{IR}^{7}$ might associated with gastroesophageal reflux disease (GERD), Barrett's esophagus (BE) and EAC. ${ }^{10}$ Although epidemiologic studies do not suggest causality with $\mathrm{Hp}$, the interplay between $\mathrm{Hp}$ and host factors plays an important role in the pathogenesis of GERD and its complications BE and EAC in certain subpopulations. ${ }^{10}$

Therefore, casting further light in the uncertain pathophysiological mechanisms underlying $\mathrm{Hp}$ and CRN association, apart from gastrin mitogenic action mentioned by the aurhors, ${ }^{1}$ our results indicate that $\mathrm{Hp}$-I has an impact on colorectal oncogenesis by: causing a possible chronic inflammatory mucosal damage, comparable to upper gastrointestinal tract (UGT); stimulating CSCs or recruiting BMDSCs, similar to UGT Hp-I-associated

Correspondence to: Jannis Kountouras

Department of Gastroenterology, Second Medical Clinic, Ippokration Hospital, Aristotle University of Thessaloniki, 8 Fanariou St, Byzantio, Thessaloniki 551 33, Greece

Tel: +30-2310-892238, Fax: +30-2310-992794, E-mail: jannis@auth.gr

Received on August 6, 2016. Revised on February 13, 2017. Accepted on March 3, 2017. Published online July 7, 2017

pISSN 1976-2283 eISSN 2005-1212 https://doi.org/10.5009/gnl16389

@) This is an Open Access article distributed under the terms of the Creative Commons Attribution Non-Commercial License (http://creativecommons.org/licenses/by-nc/4.0) which permits unrestricted non-commercial use, distribution, and reproduction in any medium, provided the original work is properly cited. 
chronic inflammation, AG, hyperplasia, metaplasia, dysplasia and BMDSCs recruitment that may facilitate tumor formation and progression in animal models and humans; $;{ }^{5,6}$ and affecting MetS parameters, oncogenes and immune surveillance processes, that may be involved in the sequence: colon epithelium transformation to CRA-dysplasia-CRC development/progression. Finally, $H p$-related IR and additional MetS parameters might also contribute to GERD-BE-EAC sequence particularly in certain subpopulations, ${ }^{10}$ though further studies are warranted to elucidate the proposed mechanisms involved in $\mathrm{Hp}$-induced colorectal and possibly UGT oncogenesis.

Therefore, Hp and MetS-related colon oncogenesis might justify CRN screening and surveillance program in the mentioned by the authors and by others high-risk patients ${ }^{9}$ and Hp eradication might inhibit the development or delay progression of CRN and possibly EAC.

\section{CONFLICTS OF INTEREST}

No potential conflict of interest relevant to this article was reported.

\section{REFERENCES}

1. Lee JY, Park HW, Choi JY, et al. Helicobacter pylori infection with atrophic gastritis is an independent risk factor for advanced colonic neoplasm. Gut Liver 2016;10:902-909.

2. Inoue I, Kato J, Tamai H, et al. Helicobacter pylori-related chronic gastritis as a risk factor for colonic neoplasms. World J Gastroenterol 2014;20:1485-1492.

3. Pilpilidis I, Kountouras J, Zavos C, Katsinelos P. Upper gastrointestinal carcinogenesis: H. pylori and stem cell cross-talk. J Surg Res 2011;166:255-264.

4. Kapetanakis N, Kountouras J, Zavos C, et al. Re: Helicobacter pylori infection and colorectal cancer risk: evidence from a large population-based case-control study in Germany. Am J Epidemiol 2012;176:566-567.

5. Kapetanakis N, Kountouras J, Zavos C, et al. Association of Helicobacter pylori infection with colorectal cancer. Immunogastroenterology 2013;2:47-56.

6. Kountouras J, Kapetanakis N, Zavos C, et al. Impact of Helicobacter pylori infection on colon oncogenesis. Am J Gastroenterol 2013;108:625-626.

7. Polyzos SA, Kountouras J, Zavos C, Deretzi G. Helicobacter pylori infection and insulin resistance. Helicobacter 2013;18:165-166.

8. Polyzos SA, Kountouras J, Papatheodorou A, et al. Helicobacter pylori infection in patients with nonalcoholic fatty liver disease. Metabolism 2013;62:121-126.

9. Romiopoulos I, Kountouras J, Polyzos SA, et al. Helicobacter pylori-related metabolic syndrome might justify earlier colorectal cancer screening. Gastrointest Endosc 2014;80:188-189.

10. Kountouras J, Polyzos SA, Zeglinas C, et al. Helicobacter pylorirelated metabolic syndrome as predictor of progression to esophageal carcinoma in a subpopulation-based Barrett's esophagus cohort. Gastrointest Endosc 2017;85:462-463. 